\title{
Surface plasmon polariton absorption modulator
}

\author{
A. Melikyan, ${ }^{1, *}$ N. Lindenmann, ${ }^{1}$ S. Walheim, ${ }^{3,5}$ P. M. Leufke, ${ }^{3}$ S. Ulrich,${ }^{4}$ J. Ye, ${ }^{4}$ \\ P. Vincze, ${ }^{3}$ H. Hahn, ${ }^{3}$ Th. Schimmel, ${ }^{3,5}$ C. Koos, ${ }^{1,2}$ W. Freude, ${ }^{1,2}$ and J. Leuthold ${ }^{1,2}$ \\ ${ }^{1}$ Institute of Photonics and Quantum Electronics, Karlsruhe Institute of Technology, 76131 Karlsruhe, Germany \\ ${ }^{2}$ Institute of Microstructure Technology, Karlsruhe Institute of Technology, \\ 76344 Eggenstein-Leopoldshafen, Germany \\ ${ }^{3}$ Institute of Nanotechnology, Karlsruhe Institute of Technology, 76344 Eggenstein-Leopoldshafen, Germany \\ ${ }^{4}$ Institute for Materials Research I, Karlsruhe Institute of Technology, 76344 Eggenstein-Leopoldshafen, Germany \\ ${ }_{5}^{5}$ Institute of Applied Physics, Karlsruhe Institute of Technology, 76131 Karlsruhe, Germany \\ *argishti.melikyan@kit.edu
}

\begin{abstract}
An electrically controlled ultra-compact surface plasmon polariton absorption modulator (SPPAM) is proposed. The device can be as small as a few micrometers depending on the required extinction ratio and the acceptable loss. The device allows for operation far beyond $100 \mathrm{Gbit} / \mathrm{s}$, being only limited by $R C$ time constants. The absorption modulator comprises a stack of metal / insulator / metal-oxide / metal layers, which support a strongly confined asymmetric surface plasmon polariton (SPP) in the 1.55 $\mu \mathrm{m}$ telecommunication wavelength window. Absorption modulation is achieved by electrically modulating the free carrier density in the intermediate metal-oxide layer. The concept is supported by proof-of-principle experiments.
\end{abstract}

(C) 2011 Optical Society of America

OCIS codes: (250.5403) Plasmonics; (240.6680) Surface plasmons; (250.4110) Modulators; (250.7360) Waveguide modulators; (230.2090) Electro-optical devices.

\section{References and links}

1. H. Fukano, T. Yamanaka, M. Tamura, and Y. Kondo, "Very-low-driving-voltage electroabsorption modulators operating at $40 \mathrm{~Gb} / \mathrm{s}$," J. Lightwave Technol. 24(5), 2219-2224 (2006).

2. Y. Chiu, S. Z. Zhang, V. Kaman, J. Piprek, and J. E. Bowers, "High-speed traveling-wave electro-absorption modulators," Proc. SPIE 4490, 1-10 (2001).

3. G. T. Reed, G. Mashanovich, F. Y. Gardes, and D. J. Thomson, "Silicon optical modulators," Nat. Photonics 4(8), 518-526 (2010).

4. J. Leuthold, C. Koos, and W. Freude, "Nonlinear silicon photonics," Nat. Photonics 4(8), 535-544 (2010).

5. L. Liao, A. Liu, D. Rubin, J. Basak, Y. Chetrit, H. Nguyen, R. Cohen, N. Izhaky, and M. Paniccia, "40 Gbit / s silicon optical modulator for highspeed applications," Electron. Lett. 43(22), 1196-1197 (2007).

6. L. Alloatti, D. Korn, D. Hillerkuss, T. Vallaitis, J. Li, R. Bonk, R. Palmer, T. Schellinger, A. Barklund, R. Dinu, J. Wieland, M. Fournier, J. Fedeli, W. Bogaerts, P. Dumon, R. Baets, C. Koos, W. Freude, and J. Leuthold, "Silicon high-speed electro-optic modulator," in Group IV Photonics, p. ThC2 (2010).

7. W. Freude, J. Leuthold, L. Alloatti, T. Vallaitis, D. Korn, R. Palmer, C. Koos, J. Brosi, P. Dumon, R. Baets, M. Scimeca, I. Biaggio, B. Breiten, F. Diederich, A. Barklund, R. Dinu, and J. Wieland, " 100 Gbit / s electro-optic modulator and $56 \mathrm{Gbit} / \mathrm{s}$ wavelength converter for DQPSK data in silicon-organic hybrid (SOH) technology," in Photonics Society Summer Topical Meeting Series, 2010 IEEE, pp. $96-97$ (2010).

8. Q. Xu, B. Schmidt, S. Pradhan, and M. Lipson, "Micrometre-scale silicon electro-optic modulator," Nature 435(7040), 325-327 (2005).

9. T. Nikolajsen, K. Leosson, and S. I. Bozhevolnyi, "Surface plasmonpolariton based modulators and switches operating at telecom wavelengths," Appl. Phys. Lett. 85(24), 5833-5835 (2004).

10. J. A. Dionne, K. Diest, L. A. Sweatlock, and H. A. Atwater, "PlasMOStor: a metal-oxide-Si field effect plasmonic modulator," Nano Lett. 9(2), 897-902 (2009).

11. S.-I. Inoue and S. Yokoyama, "Numerical simulation of ultra-compact electro-optic modulator based on nanoscale plasmon metal gap waveguides," Electron. Lett. 45(21), 1087-1089 (2009).

12. C. Jung, S. Yee, and K. Kuhn, "Electro-optic polymer light modulator based on surface plasmon resonance," Appl. Opt. 34(6), 946-949 (1995).

13. C. Jung, S. Yee, and K. Kuhn, "Integrated optics waveguide modulator based on surface plasmon resonance," J. Lightwave Technol. 12(10), 1802-1806 (1994).

14. W.-K. Kuo and M.-T. Chen, "Simulation study of surface-plasmon-resonance electro-optic light modulator based on a polymer grating coupler," Opt. Lett. 34(24), 3812-3814 (2009).

\#143707 - \$15.00 USD Received 7 Mar 2011; revised 14 Apr 2011; accepted 16 Apr 2011; published 21 Apr 2011

(C) 2011 OSA

25 April 2011 / Vol. 19, No. 9 / OPTICS EXPRESS 8855 
15. Z. Wu, R. L. Nelson, J. W. Haus, and Q. Zhan, "Plasmonic electro-optic modulator design using a resonant metal grating," Opt. Lett. 33(6), 551-553 (2008).

16. J. S. Schildkraut, "Long-range surface plasmon electrooptic modulator," Appl. Opt. 27(21), 4587-4590 (1988).

17. O. Solgaard, F. Ho, J. I. Thackara, and D. M. Bloom, "High frequency attenuated total internal reflection light modulator," Appl. Phys. Lett. 61(21), 2500-2502 (1992).

18. M. J. Dicken, L. A. Sweatlock, D. Pacifici, H. J. Lezec, K. Bhattacharya, and H. A. Atwater, "Electrooptic modulation in thin film barium titanate plasmonic interferometers," Nano Lett. 8(11), 4048-4052 (2008).

19. A. Melikyan, T. Vallaitis, N. Lindenmann, T. Schimmel, W. Freude, and J. Leuthold, "A surface plasmon polariton absorption modulator," in Conference on Lasers and Electro-Optics, p. JThE77 (2010).

20. E. Feigenbaum, K. Diest, and H. A. Atwater, "Unity-order index change in transparent conducting oxides at visible frequencies," Nano Lett. 10(6), 2111-2116 (2010).

21. B. Prade, J. Y. Vinet, and A. Mysyrowicz, "Guided optical waves in planar heterostructures with negative dielectric constant," Phys. Rev. B Condens. Matter 44(24), 13556-13572 (1991).

22. J. J. Burke, G. I. Stegeman, and T. Tamir, "Surface-polariton-like waves guided by thin, lossy metal films," Phys. Rev. B Condens. Matter 33(8), 5186-5201 (1986).

23. M. L. Nesterov, A. V. Kats, and S. K. Turitsyn, "Extremely short-length surface plasmon resonance devices," Opt. Express 16(25), 20227-20240 (2008).

24. F. Neumann, Y. A. Genenko, C. Melzer, S. V. Yampolskii, and H. von Seggern, "Self-consistent analytical solution of a problem of charge-carrier injection at a conductor/insulator interface," Phys. Rev. B 75(20), 205322 (2007).

25. F. Michelotti, L. Dominici, E. Descrovi, N. Danz, and F. Menchini, “Thickness dependence of surface plasmon polariton dispersion in transparent conducting oxide films at 1.55 microm," Opt. Lett. 34(6), 839-841 (2009).

26. S. Dasgupta, M. Lukas, K. Dössel, R. Kruk, and H. Hahn, "Electron mobility variations in surface-charged indium tin oxide thin films," Phys. Rev. B 80(8), 085425 (2009).

27. P. B. Johnson and R. W. Christy, “Optical constants of the noble metals,” Phys. Rev. B 6(12), 4370-4379 (1972).

28. C. Rhodes, M. Cerruti, A. Efremenko, M. Losego, D. E. Aspnes, J.-P. Maria, and S. Franzen, "Dependence of plasmon polaritons on the thickness of indium tin oxide thin films," J. Appl. Phys. 103(9), 093108 (2008).

29. H. S. Kwok, X. W. Sun, and D. H. Kim, "Pulsed laser deposited crystalline ultrathin indium tin oxide films and their conduction mechanisms," Thin Solid Films 335(1-2), 299-302 (1998).

\section{Introduction}

Optical modulators in future integrated circuits should enable data rates of $100 \mathrm{Gbit} / \mathrm{s}$ and beyond; they should be compact with footprints comparable to those of electronic devices (typically $<1 \mu \mathrm{m}^{2}$ ), and fabrication with standard CMOS technology should be possible. Electro-absorption modulators (EAM) belong to the most compact commercially available data encoding devices in optical communications. They are available in typical lengths of $200 \mu \mathrm{m}$, supporting data rates of $40 \mathrm{Gbit} / \mathrm{s}$ [1]. Analog bandwidths can be as high as $100 \mathrm{GHz}$ for traveling wave structures [2]. Unfortunately, high-speed EAM usually rely on costly InP technology, which is challenging for economical mass production.

Besides EAMs, more recently, CMOS-compatible silicon-based solutions have been suggested $[3,4]$. High speed all-silicon and silicon organic hybrid (SOH) modulators with lengths of a few millimeters and speeds exceeding $40 \mathrm{Gbit} / \mathrm{s}$ have already been demonstrated [5-7]. More compact Si-based modulators have been demonstrated in resonant structures. These devices exploit free-carrier dispersion either in a forward or reverse-biased pn junction [3,8], allowing for operation at $10 \mathrm{Gbit} / \mathrm{s}$. The operating speeds of such devices are ultimately limited by the finite mobility of free carriers. Thus, an ultra-fast, ultra-compact modulator based on a CMOS compatible technology has yet to be developed.

This can be achieved by using nano-plasmonic structures that enable strong confinement of both electrical and optical fields, thereby enhancing interaction with the underlying material and hence the modulation of the optical signal. Several approaches have been proposed for electro-optical manipulations of surface plasmon polaritons (SPPs). A technique proposed by Nikolajsen et al. [9] exploits the temperature-dependence of the refractive index in benzocyclobutene, an effect with limited operating speed. The device by Dionne et al. [10] - the socalled PlasMOStor device - exploits the carrier-induced refractive index change in a silicon layer. While this device works well, its speed is again limited by the electron mobility in the $170 \mathrm{~nm}$ thick active silicon layer. Since early in the 1980s, various approaches have been proposed for SPP modulation employing the linear electro-optic effect, e.g. in a polymer sandwiched between two metallic patches [11-18]. The feasibility of this concept is still to be

\#143707 - \$15.00 USD Received 7 Mar 2011; revised 14 Apr 2011; accepted 16 Apr 2011; published 21 Apr 2011

(C) 2011 OSA

25 April 2011 / Vol. 19, No. 9 / OPTICS EXPRESS 8856 
investigated. Recently, a new modulation technique has been reported by our group [19] and independently by Feigenbaum et al. [20]. The technique relies on carrier modulation in a metal-oxide layer guiding a SPP. The approach of Feigenbaum et al. exploits the phase modulation of SPP in the visible wavelength range by a voltage-induced modulation of the carrier density in a bulk ITO layer. The group has reported ellipsometry measurements in the wavelength range $500 \mathrm{~nm} . .800 \mathrm{~nm}$ that show significant changes of the voltage-dependent refractive index in bulk ITO layers that are transparent at these wavelengths. In contrast, our approach is based on a change of the absorption experienced by an SPP at $1.55 \mu \mathrm{m}$ telecommunication wavelength. The absorption change is again caused by a voltage-induced carrier density modulation in ITO [19]. The advantage of our approach is that it has the capability of operating at telecommunication wavelengths, and that it is potentially free from carrier-related speed limitations.

In this paper, we provide in-detail simulations and give first experimental evidence of the feasibility of an ultra-compact, CMOS compatible plasmonic SPPAM operated by directly modulating the electron carrier density in a metal-oxide layer. The suggested modulator operates at speeds that are only limited by the electronic drive circuits. To the best of our knowledge this is the first conceptual report of such an SPPAM supported by measurements. Unlike many prior plasmon modulator concepts, our approach is well suited for an operation in the near infrared and in particular in the telecommunication window around $1.3 \mu \mathrm{m}$ and $1.55 \mu \mathrm{m}$, i.e. at a wavelength range where the plasmon resonances at the conventional metal/insulator interfaces are broad and therefore cannot be used for efficient switching.

The paper is organized as follows: In Section 2, we introduce a design of the SPPAM compatible with the standard silicon-on-insulator (SOI) platform. In Section 3, the dispersion relation (DR) for the suggested four-layer configuration is derived in general. Further, the properties of the carrier accumulation layer are calculated by means of the Thomas-Fermi approximation, and an in-depth study of the DR for a particular case is given in Section 3. Section 4 is devoted to the description of recent experimental results which validate the SPP absorption modulation concept.

\section{Modulator design}

The configuration of the suggested SPPAM is given in Fig. 1. It comprises a silicon strip waveguide on a buried silicon-oxide layer. The central plasmonic section consists of a dielectric layer and a thin metal-oxide layer sandwiched between highly conductive metal layers. The metal layers serve as SPP waveguides and as electrodes for applying an electric field across the dielectric.

The operation principle of the modulator is as follows. An optical quasi-TM mode featuring a dominant vertical electric field component is excited in the silicon strip waveguide (Fig. 1 , blue blocks). The light is subsequently coupled to the plasmonic waveguide, see the electric field magnitudes in Fig. 1 insets (a)-(c). The illustrative numbers for the following description were taken from Table 1 in Appendix A.1. Applying an electric field of, e.g., $10 \mathrm{~V} / 30 \mathrm{~nm}$ changes the free carrier density in the intermediate metal-oxide layer (e.g., $8 \mathrm{~nm}$ thick ITO) from $N_{0}=9.25 \times 10^{26} \mathrm{~m}^{-3}$ to $9.34 \times 10^{26} \mathrm{~m}^{-3}$, thereby changing its complex permittivity from $\varepsilon=-1.667+0.824 \mathrm{i}$ to $-1.721+0.832 \mathrm{i}$. As a consequence, the absorption experienced by the SPP is decreased from $\alpha=2.19 \mu \mathrm{m}^{-1}$ to $2.07 \mu \mathrm{m}^{-1}$. After propagating through the active plasmonic section of length $L$ (e. g., $1 \mu \mathrm{m}$ ), the plasmonic mode couples back to the photonic mode inside the output coupling zone. As an overall result, the applied electric field modulates the output power.

In this work we have chosen for the active plasmonic section a sequence of four layers: From top to bottom (inset Fig. 1) we have a silver (Ag) layer, a thin metal-oxide layer, here indium tin oxide (ITO), a silicon dioxide $\left(\mathrm{SiO}_{2}\right)$ film and another silver layer. The whole structure is embedded in either PMMA or $\mathrm{SiO}_{2}$. The choice of the dielectric sandwiched be-

\#143707 - \$15.00 USD Received 7 Mar 2011; revised 14 Apr 2011; accepted 16 Apr 2011; published 21 Apr 2011

(C) 2011 OSA 25 April 2011 / Vol. 19, No. 9 / OPTICS EXPRESS 8857 
tween two electrodes is based on studies of the dispersion relation introduced below. The device length can be in the range of a $1 \mu \mathrm{m}$ up to a few micrometers, depending on the requirements with respect to the extinction ratio and the acceptable insertion loss.

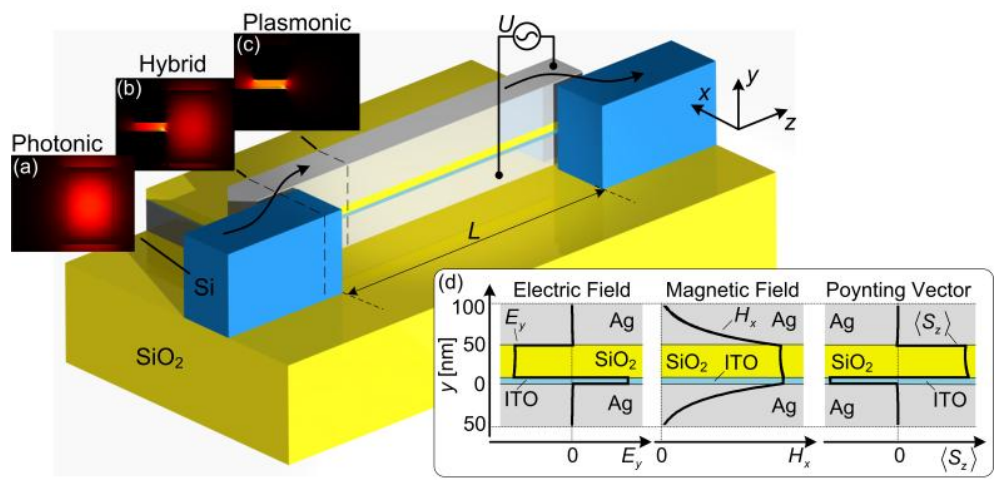

Fig. 1. The structure of a surface plasmon polariton absorption modulator (SPPAM). Light is coupled from a silicon nanowire into an active plasmonic section by means of a directional coupler. The active section consists of a stack of silver ( $\mathrm{Ag}$ ), indium tin oxide (ITO), and $\mathrm{SiO}_{2}$ layers. The absorption coefficient of the SPP is modulated by applying a voltage between the two silver electrodes. The insets show how a photonic mode (a) in a silicon strip waveguide excites a SPP (c) via a hybrid mode (b) in directional coupler. The insets in (d) show the electric field $E_{\mathrm{y}}$ and the magnetic field $H_{\mathrm{x}}$ as well as the time-averaged Poynting vector distributions $\left\langle S_{z}\right\rangle=\operatorname{Re}\{E \times H\} / 2$ in the active plasmonic part. The plot of the Poynting vector shows the power confinement of the SPP in the ITO layer. The length $L$ describes the size of the modulator along the light propagation direction.

The characteristics of ITO are key to the operation of the suggested structure. On the one hand ITO features a metallic behavior with relatively high carrier mobility, on the other hand, the carrier density is low enough to result in a small real part of the dielectric permittivity and a large electron screening length. This provides the following advantages for the operation of the modulator:

- There are no carrier-related speed limitations because of the high carrier mobility in ITO.

- The small magnitude of the complex permittivity of ITO compared to noble metals and typical dielectrics provides strong field enhancement within the ITO layer for the wavelength range of interest. Simulations in Fig. 1 inset (d) show that the electric field in ITO is large, while there is hardly any field within the silver layer. If $\mathrm{SiO}_{2}$ was replaced by $\mathrm{Si}_{3} \mathrm{~N}_{4}$, the field confinement in the ITO layer could be further enhanced.

- The overlap between the externally induced charges and the SPP mode is particularly good in ITO compared to other metals because of the relatively large electron screening length. The large overlap is important to exploit the plasma-induced absorption change.

- Because in our case the SPP represents a field confined to an area far below the diffraction limit, a relatively small voltage creates an enormous electric field in the dielectric layer providing the necessary amount of carrier change in the ITO layer.

As a result of this design optimization, the size of the device can be as small as a few hundred nanometers in transverse direction by a few micrometers in length, a range which does not influence the modulator speed. In the future, such techniques could allow integrating hundreds of modulators on the same chip.

\#143707 - \$15.00 USD Received 7 Mar 2011; revised 14 Apr 2011; accepted 16 Apr 2011; published 21 Apr 2011

(C) 2011 OSA

25 April 2011 / Vol. 19, No. 9 / OPTICS EXPRESS 8858 


\section{Theory}

For discussing the basic operation principles of a plasmonic absorption modulator, we first investigate a $2 \mathrm{D}$ slab structure. We then identify key characteristic quantities, namely dispersion relation, insertion loss and extinction ratio, and study them in view of an actual 3D design. Finally, we specify the 3D SPP modal field and the propagation constant.

\subsection{Dispersion relation of the SPP in a four-layer slab configuration}

We derive here the dispersion relation (DR) of a four-layer active plasmonic structure, and we follow the procedure as described formerly [21-23]. The geometry of the problem and the respective permittivities are shown in Figs. 2(a) and 2(b). An SPP exist only in TM polarization, i.e. when the magnetic field is parallel to the metal-dielectric boundaries, see Fig. 2. Therefore we solve the wave equation for TM modes only.

(a)

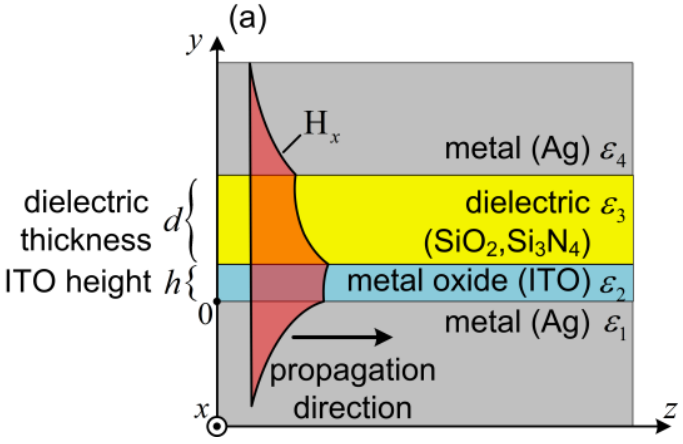

(b)

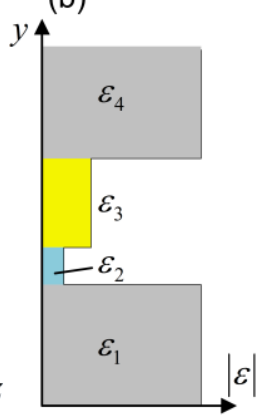

(c)

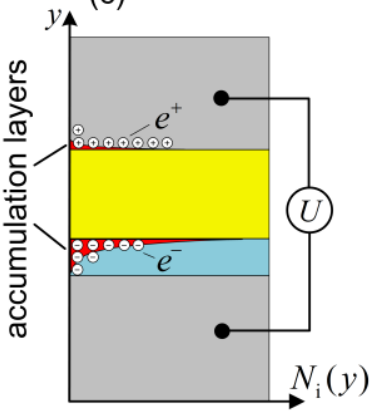

Fig. 2. Plasmonic structure with metal/dielectric/metal-oxide/metal layers. (a) Geometry and (b) dielectric permittivity distribution. The $H_{x}$ component of the SPP magnetic field is schematically shown as a contour filled with reddish color in (a). The SPP propagates along the positive $z$-direction. (c) Carrier density distributions $N_{\mathrm{i}}(y)$ in both electrodes.

Assuming a time and $z$-coordinate dependency $e^{-i(\omega t-\beta z)}$ for the magnetic field $H_{x}$, the wave equation takes the form

$$
\frac{d^{2} H_{x}(y)}{d y^{2}}+\left[k_{0}^{2} \varepsilon(y)-\beta^{2}\right] H_{x}(y)=0,
$$

where $k_{0}=\omega / c$ is the magnitude of the wave vector of plane wave in free space, $\varepsilon(y)$ is the permittivity as a function of the $y$-coordinate and $\beta$ is the propagation constant of the SPP. Without loss of generality we assume that the magnetic field $H_{x}$ is confined in the dielectric and metal oxide layers and decays exponentially in the adjacent Ag layers. Thus, the solution of Eq. (1) satisfying the above requirements in each layer can be written with the transverse propagation constant $k_{m}=\sqrt{\varepsilon_{m} k_{0}^{2}-\beta^{2}}$ as

$$
\begin{aligned}
& H_{x}(y)=A_{1} \mathrm{e}^{i k_{1} y} \quad \text { for }-\infty<y<0 \\
& H_{x}(y)=A_{2} \mathrm{e}^{i k_{2} y}+B_{2} \mathrm{e}^{-i k_{2} y} \quad \text { for } \quad 0<y<h \\
& H_{x}(y)=A_{3} \mathrm{e}^{i k_{3}(y-h)}+B_{3} \mathrm{e}^{-i k_{3}(y-h)} \text { for } \quad h<y<h+d \\
& H_{x}(y)=B_{4} \mathrm{e}^{-i k_{4}(y-d-h)} \quad \text { for } h+d<y<+\infty
\end{aligned}
$$

The subscripts $m=1$ and $m=4$ are used for the bottom and top metal layers, the subscript $m=$ 2 denotes the metal oxide layer, and the subscript $m=3$ is assigned to the dielectric layer. The constants in front of the exponential functions describe the magnetic field amplitude in the 
respective medium. The electric field in each material can then be derived employing Maxwell's equations ( $\varepsilon_{0}$ is the vacuum permittivity):

$$
\begin{aligned}
E_{z}^{m} & =-i \frac{1}{\omega \varepsilon_{0} \varepsilon_{m}} \frac{\partial H_{x}^{m}}{\partial y} \\
E_{y}^{m} & =-\frac{\beta}{\omega \varepsilon_{0} \varepsilon_{m}} H_{x}^{m}
\end{aligned}
$$

Applying the boundary condition for the tangential components of the magnetic and the electric field allows to derive the dispersion relation

$$
\frac{1+r_{12} \mathrm{e}^{-i 2 k_{2} h}}{1-r_{12} \mathrm{e}^{-i 2 k_{2} h}}=\frac{k_{2} \varepsilon_{3}}{k_{3} \varepsilon_{2}}\left[\frac{r_{34} \mathrm{e}^{-i 2 k_{3} d}+1}{r_{34} \mathrm{e}^{-i 2 k_{3} d}-1}\right],
$$

where $r_{12}=\left(k_{2} \varepsilon_{1}-k_{1} \varepsilon_{2}\right) /\left(k_{2} \varepsilon_{1}+k_{1} \varepsilon_{2}\right)$ and $r_{34}=\left(k_{3} \varepsilon_{4}-k_{4} \varepsilon_{3}\right) /\left(k_{3} \varepsilon_{4}+k_{4} \varepsilon_{3}\right)$. The solutions of Eq. (4) represent the eigenmodes of such a four-layer structure with their respective complex eigenvalues $\beta$. The imaginary part of $\beta$ describes the absorption in the system, and the power absorption coefficient is defined as $\alpha=2 \operatorname{Im}[\beta]$.

The absorption modulator performance needs to be optimized for the lowest overall losses and the highest possible extinction ratio. Therefore it is convenient to introduce two parameters, namely the propagation length $L_{e}$ and the length $L_{\mathrm{ldB}}$. The effective propagation length

$$
L_{e}=\frac{1}{\alpha}=(2 \operatorname{Im}[\beta])^{-1}
$$

is the length after which the intensity of the SPP has decreased to a fraction of 1 / e of the initial intensity.

In addition we define the $1 \mathrm{~dB}$ on-off length $L_{\mathrm{ldB}}$ where the on-off ratio is $\mathrm{ER}=1 \mathrm{~dB}$ for an (arbitrarily chosen) DC on-voltage of $U=10 \mathrm{~V}$ which is applied across the electrodes. The logarithmic extinction ratio ER is defined as

$$
\begin{aligned}
\mathrm{ER}=10 \lg \left(P_{\text {on }} / P_{\text {off }}\right) & =10 \lg \left(P_{0} \mathrm{e}^{-\alpha_{\text {on }} L} / P_{0} \mathrm{e}^{-\alpha_{\text {off }} L}\right) \\
& =10 \lg \left(\mathrm{e}^{\alpha_{\text {off }}-\alpha_{\text {on }} L}\right)=4.34 \times\left|\alpha_{\text {off }}-\alpha_{\text {on }}\right| L^{.}
\end{aligned}
$$

The logarithmic extinction ratio depends linearly on the difference of the absorption coefficients $\Delta \alpha_{10 \mathrm{v}}=\left|\alpha_{\text {off }}-\alpha_{\text {on }}\right|$ for the voltage-off and the voltage-on states. The relation between $L_{\mathrm{ldB}}$ and the $1 \mathrm{~dB}$ extinction ratio is

$$
\mathrm{ER}_{\mathrm{ldB}}=1 \mathrm{~dB}, \quad L_{\mathrm{ldB}}=\frac{1}{4.34 \times \Delta \alpha_{10 \mathrm{v}}} .
$$

To calculate the absorption coefficient in the voltage-on and voltage-off states the Thomas-Fermi screening theory is employed as discussed in the next section.

Finally, we introduce a figure of merit (FoM) that relates the effective propagation length $L_{\mathrm{e}}$ to the $1 \mathrm{~dB}$ on-off length $L_{\mathrm{ldB}}$. The FoM quantitatively describes the extinction ratio achievable in a device of length $L_{\mathrm{e}}$ operated with a $10 \mathrm{~V}$ voltage,

$$
\mathrm{FoM}=L_{\mathrm{e}} / L_{\mathrm{ldB}} \text {. }
$$

The larger the FoM, the better the performance is, i.e. the lower the propagation losses are for a $1 \mathrm{~dB}$ extinction ratio and an applied on-voltage of $10 \mathrm{~V}$.

\#143707 - \$15.00 USD Received 7 Mar 2011; revised 14 Apr 2011; accepted 16 Apr 2011; published 21 Apr 2011 (C) 2011 OSA 25 April 2011 / Vol. 19, No. 9 / OPTICS EXPRESS 8860 


\subsection{Thomas-Fermi screening theory}

An external voltage applied to the metallic electrodes changes the carrier density in the metaloxide layer and hence influences the absorption coefficient of the SPP. For a proper estimation of this effect we employ the Thomas-Fermi screening theory for deriving the carrier distribution for a given voltage applied to the electrodes. The resulting spatial carrier distribution can then be used for modeling the complex dielectric permittivity of the metal-oxide layer via the Drude model, which then yields the complete information necessary for solving Eq. (4) for the on-state.

The Poisson equation relates the electric potential $\phi(y)$ to the induced charge density $N_{\mathrm{i}}(y)$ within the ITO layer,

$$
\Delta \phi(y)=\frac{e\left(N_{\mathrm{i}}(y)-N_{0}\right)}{\varepsilon_{0} \varepsilon_{\mathrm{ITO}}} .
$$

Here, $e$ is the elementary charge, $\varepsilon_{0}$ is the vacuum permittivity, $\varepsilon_{\text {Іто }}=9.3$ is the relative static permittivity of ITO [24], and $N_{0}$ is the bulk free carrier density of ITO.

Following the Thomas-Fermi approach the total free carrier density and the potential are related by

$$
N_{\mathrm{i}}(y)=\frac{1}{3 \pi^{2}}\left(\frac{8 \pi^{2} m_{\mathrm{eff}}}{h^{2}}\right)^{3 / 2}\left(E_{F}+e \phi(y)\right)^{3 / 2},
$$

where the Fermi energy is defined as

$$
E_{F}=\left(\frac{h^{2}}{8 \pi^{2} m_{\mathrm{eff}}}\left[3 \pi^{2} N_{0}\right]^{2 / 3}\right) .
$$

In the latter expression $h$ is Planck's constant. The electron effective mass $m_{\text {eff }}$ for ITO is given in Table 1 of Appendix A in terms of the free electron mass $m_{e}=9.1 \times 10^{-31} \mathrm{~kg}$. The bulk free carrier density $N_{0}$ of ITO can be calculated from the plasma frequency $\omega_{\mathrm{p}}$ in Table 1 via the relation $N_{0}=\varepsilon_{0} m_{\text {eff }} \omega_{\mathrm{p}} / e^{2}$.

In the Thomas-Fermi approximation, Eq. (9) and Eq. (10) have been solved analytically for the case of a weak potential $\left(e \phi<<E_{F}\right)$ resulting in the so-called Thomas-Fermi screening length $\lambda_{\mathrm{TF}}=\left[\frac{\varepsilon_{\text {Iто }} \varepsilon_{0} h^{2}}{4 \pi^{2} m_{\mathrm{eff}} e^{2}}\right]^{1 / 2}\left[\frac{\pi^{4}}{3 N_{0}}\right]^{1 / 6}$, which gives an estimate of the carrier density distribution at the conductor interface. The Thomas-Fermi screening length for ITO is estimated to be $\lambda_{\mathrm{TF}} \approx 6 \AA$, i.e. it is comparable to the value reported by Neumann et al. [24]. Obviously, due to the low free-carrier density of ITO, the estimated $\lambda_{\mathrm{TF}}$ is significantly larger for ITO than for noble metals where it is usually in the range of $1 \ldots 2 \AA$.

Rather than employing the weak potential approximation, we numerically solve Eq. (9) with Eqs. (10) and (11) for the ITO/dielectric interface with mixed boundary conditions

$$
\begin{gathered}
\frac{U}{d}=\left.\varepsilon_{\text {ins }} \frac{d \phi}{d y}\right|_{y \rightarrow+\mathrm{h}}=\left.\varepsilon_{\text {ITO }} \frac{d \phi}{d y}\right|_{y \rightarrow-h}, \\
\phi(y) \rightarrow 0 \text { for } y \rightarrow-\infty,
\end{gathered}
$$

\#143707 - \$15.00 USD Received 7 Mar 2011; revised 14 Apr 2011; accepted 16 Apr 2011; published 21 Apr 2011

(C) 2011 OSA 25 April 2011 / Vol. 19, No. 9 / OPTICS EXPRESS 8861 
where $U$ and $d$ are the voltage and the distance between the two electrodes, respectively. The quantity $\varepsilon_{\text {ins }}$ is the dielectric permittivity of the (insulating) dielectric. Due to their large dielectric strengths (high break-through voltage) silicon dioxide $\left(\mathrm{SiO}_{2}\right)$ with $\varepsilon_{\mathrm{SiO}_{2}}=3.9$ and silicon nitride $\left(\mathrm{Si}_{3} \mathrm{~N}_{4}\right)$ with $\varepsilon_{\mathrm{Si}_{3} \mathrm{~N}_{4}}=7.8$ have been considered as possible dielectrics for the above introduced structure. Inside the ITO layer, the second boundary condition is numerically approximated by taking the potential to be zero at a point many screening lengths $\lambda_{\mathrm{TF}}$ away from the ITO/dielectric interface.

Solving the boundary value problem provides the potential distribution $\phi(y)$ in the ITO layer. This is then used to calculate the induced carrier density $N_{i}(y)$ near the interface with the help of Eq. (10) and Eq. (11). In order to calculate the effect of this carrier density change on the DR it is more practical to use the average carrier density across the thin ITO layer with height $h,\left\langle N_{i}(y)\right\rangle_{h}$. This is justified by the fact that the SPP mode profile exhibits a relative constant amplitude in the ITO layer, Fig. 1. The relative change of the free carrier density in the $8 \mathrm{~nm}$ ITO layer is estimated to be $1 \%$ for a $10 \mathrm{~V}$ voltage applied across a $30 \mathrm{~nm}$ thick $\mathrm{SiO}_{2}$ layer. Below we show that this relatively small carrier density changes can be good enough for on-off switching.

\subsection{Dispersion relation of SPP for the Ag-ITO-dielectric-Ag configuration}

To study the effect of a carrier density change in the SPP guiding layers we have numerically solved the dispersion relation as derived in Eq. (4). The material dispersion of both ITO and silver are taken into account via the Drude model, see Appendix A. In the following, a value of $2.19 \mathrm{PHz}(0.46 \mathrm{PHz})$ is considered for the plasma frequency of $\mathrm{Ag}$ (ITO).

Dispersion relation and absorption coefficient of an Ag / ITO $(8 \mathrm{~nm}) / \mathrm{Si}_{3} \mathrm{~N}_{4}(70 \mathrm{~nm}) / \mathrm{Ag}$ structure (see inset Fig. 3(b)) are plotted in Fig. 3 as a function of frequency for two ITO carrier densities, $N_{0}$ and $\left\langle N_{i}(y)\right\rangle_{h}$. The quantities $N_{0}$ and $\left\langle N_{i}(y)\right\rangle_{h}$ correspond to densities without and with a voltage applied to the Ag-electrodes. For illustration, $\left\langle N_{i}(y)\right\rangle_{h}$ has been chosen to be $\left\langle N_{\mathrm{i}}(y)\right\rangle_{h}=1.05 \times N_{0}$. It can be seen that the dispersion of the SPP at the telecommunication frequency $(193.5 \mathrm{THz})$ is basically not affected by carrier density changes within the ITO layer, Fig. 3(a). However, the change of the absorption coefficient is as large as $30 \%$. Thus, the structure operates best as an absorption modulator rather than as a phase modulator.
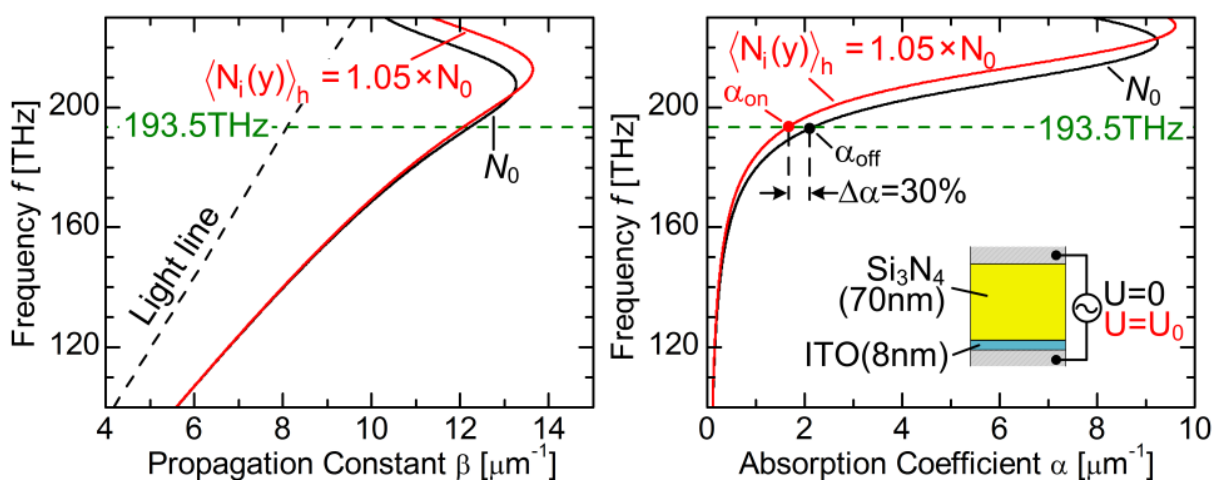

Fig. 3. Dispersion relation of SPP guided by the $\mathrm{Ag} / \mathrm{ITO}(8 \mathrm{~nm}) / \mathrm{Si}_{3} \mathrm{~N}_{4}(70 \mathrm{~nm}) / \mathrm{Ag}$ layer stack. Both, propagation constant and absorption coefficient, change when the carrier density of ITO is increased by $5 \%$ (red lines). As opposed to the propagation constant the absorption coefficient varies significantly with ITO carrier density.

\#143707 - \$15.00 USD Received 7 Mar 2011; revised 14 Apr 2011; accepted 16 Apr 2011; published 21 Apr 2011 (C) 2011 OSA 25 April 2011 / Vol. 19, No. 9 / OPTICS EXPRESS 8862 
The maximum SPP propagation constant in Fig. 3(a) defines a so-called surface plasma frequency which is close to the communication frequency at $193.5 \mathrm{THz}$ due to the small plasma frequency of ITO. Decreasing the thickness $d$ of the dielectric and increasing the ITO height $h$ cause the maximum points in the dispersion relation Fig. 3 to be shifted to the lower frequency region. As a consequence, the values of $\beta$ and $\alpha$ at the communication frequency 193.5 THz (green horizontal dashed line) increase. Bound SPPs at such a configuration exist to the right of the light line, i. e., for frequencies below $240 \mathrm{THz}$ (or significantly above 240 $\mathrm{THz}$ [20] where the metallic property of ITO is less pronounced). The SPPs at the frequency range below $240 \mathrm{THz}$ are well confined to the thin ITO layer because of its semi-metallic character. Therefore, the SPP absorption coefficient reacts very sensitively to a change of the free carrier density in ITO. This allows to efficiently modulate the intensity of light by electrically modulating the carrier density in the metal oxide layer.
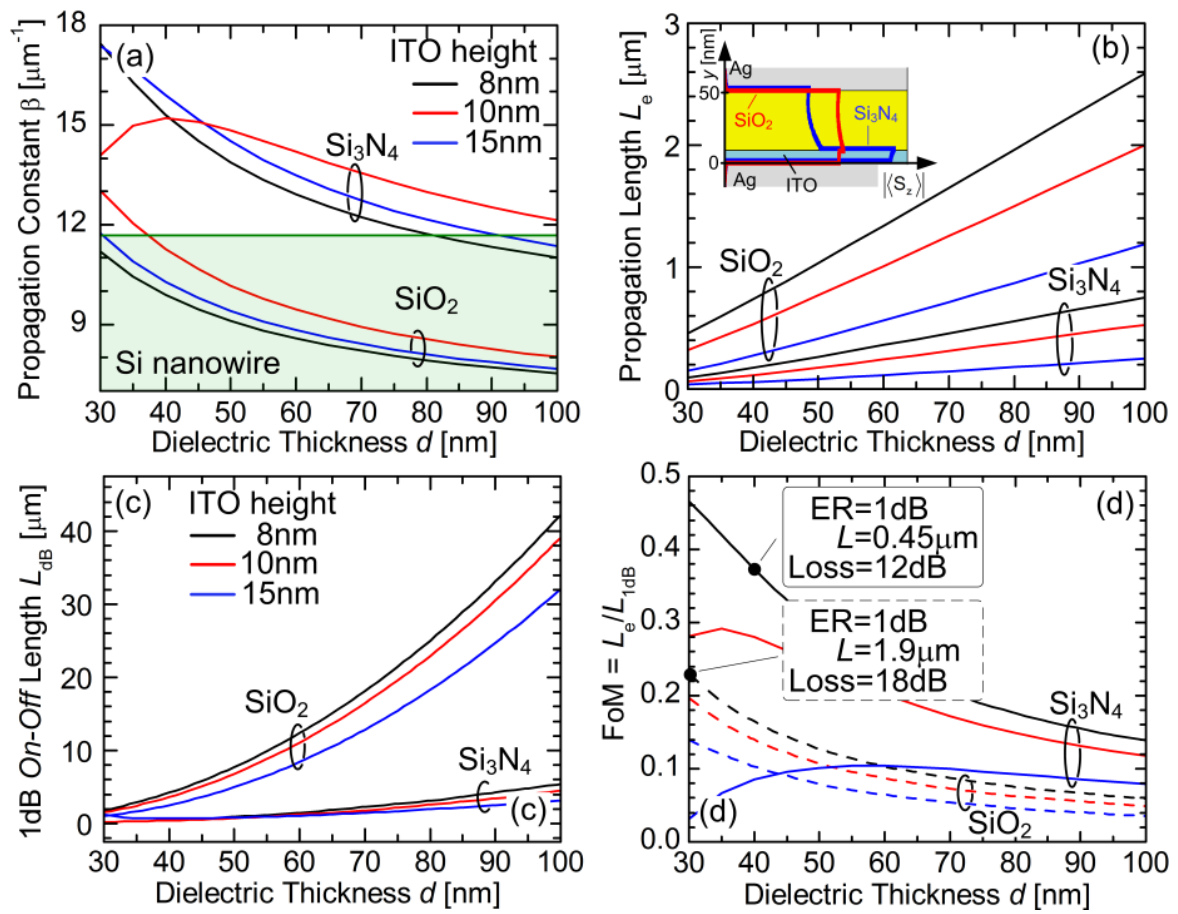

Fig. 4. Dependence of characteristic device parameters as a function of the thickness $d$ of the dielectric for two dielectric materials $\left(\mathrm{Si}_{3} \mathrm{~N}_{4}, \mathrm{SiO}_{2}\right.$, ) and for ITO thicknesses $h=8 \mathrm{~nm}$ (black line), $10 \mathrm{~nm}$ (red line) and $15 \mathrm{~nm}$ (blue line). (a) Propagation constant $\beta$. A typical propagation constant achievable in a silicon nanowire $(500 \mathrm{~nm} \times 500 \mathrm{~nm})$ waveguide is shown as a horizontal green line. Therefore, an SPP excitation via a directional coupler is easily possible for a $\mathrm{SiO}_{2}$ dielectric, but a grating coupler is preferable for a $\mathrm{Si}_{3} \mathrm{~N}_{4}$ dielectric. (b) Propagation length $L_{\mathrm{e}}$. Increasing the refractive index of the dielectric results in a decrease of $L_{\mathrm{e}}$. The inset shows the absolute value of the time-averaged Poynting vector distributions in the structures filled with $\mathrm{Si}_{3} \mathrm{~N}_{4}$ (blue line) and $\mathrm{SiO}_{2}$ (red line). It can be seen that that the power confinement in ITO is larger than in the case of $\mathrm{Si}_{3} \mathrm{~N}_{4}$. (c) Dependence of $1 \mathrm{~dB}$ on-off length $L_{1 \mathrm{~dB}}$. It can be seen that $L_{1 \mathrm{~dB}}$ is dramatically decreasing with the thickness of the dielectric, which is a result of both the static and the optical electrical field enhancement in the structure. The larger refractive index of the $\mathrm{Si}_{3} \mathrm{~N}_{4}$ gives rise to a stronger optical field confinement in the active ITO layer, which in turn results in a shorter 1dB on-off length as well as shorter propagation length. (d) Figure of merit (FoM) as a function of the thickness of the dielectric. FoM increases for smaller dielectric thicknesses. The structure with $\mathrm{Si}_{3} \mathrm{~N}_{4}$ dielectric performs considerably better. Insets give numerical examples for extinction ratio ER, device length $L$ and loss in the system for both $\mathrm{Si}_{3} \mathrm{~N}_{4}$ and $\mathrm{SiO}_{2}$. 
The modulator performance fundamentally depends on the thickness $(d)$ and on the permittivity of the dielectric, and on the height $(h)$ of the ITO. The dependencies of the propagation constant and absorption coefficient on $h$ and $d$ are depicted in Fig. 4(a) and Fig. 4(b) for two distinct dielectric materials, namely $\mathrm{Si}_{3} \mathrm{~N}_{4}$ and $\mathrm{SiO}_{2}$ with refractive indices $\sqrt{\varepsilon_{3}}=2.0$ and $\sqrt{\varepsilon_{3}}=1.44$, respectively. It can be seen that the propagation constant $\beta$ decreases with increasing $d$. The propagation length $L_{\mathrm{e}}$ increases with $d$, because the portion of the optical power propagating in the lossy ITO layer decreases. The useful SPP propagation length does not exceed a few micrometers, which makes a proper choice of the ITO thickness crucial in the design. Further, one can see that $L_{\mathrm{e}}$ is significantly smaller for a $\mathrm{Si}_{3} \mathrm{~N}_{4}$ than for a $\mathrm{SiO}_{2}$ dielectric, which is a result of a stronger SPP field confinement in the ITO layer for the former case, see inset of Fig. 4(b). The propagation constant $\beta$ as well as the propagation length $L_{e}$ increases or decreases with increasing $d$ depending on the ITO thickness and the refractive index of the dielectric layer, parameters which define the maxima in Fig. 3(a) and Fig. 3(b). For the same reason, extrema can be seen for the $\mathrm{Si}_{3} \mathrm{~N}_{4}$-curves in Fig. 4 .

A significantly smaller $1 \mathrm{~dB}$ on-off length can be observed for the structure with a $\mathrm{Si}_{3} \mathrm{~N}_{4}$ dielectric. Therefore, for finding an optimum with respect to propagation length and $1 \mathrm{~dB}$ onoff length as well as for a fair comparison of the structures with $\mathrm{Si}_{3} \mathrm{~N}_{4}$ and $\mathrm{SiO}_{2}$ dielectric, respectively, we display in Fig. 4(d) the figure of merit from Eq. (8) in dependence of the dielectric and the ITO thicknesses. The graph in combination with the numerical values of the insets indicates that significantly improved extinction ratios with an acceptable loss can be achieved for a $\mathrm{Si}_{3} \mathrm{~N}_{4}$ dielectric with device lengths shorter than $1 \mu \mathrm{m}$.

In order to make plasmonic modulators working devices, it is preferable to couple light from a photonic silicon wire waveguide into the modulation section, see Fig. 1. However, the co-integration of plasmonics and photonics requires compact and efficient SPP excitation techniques. Several authors have proposed SPP excitation making use of a directional coupler configuration between the silicon and the plasmonic waveguide. Such coupling techniques require well-defined phase matching and a strong field overlap. It can be seen in Fig. 4(a) that phase matching is difficult with a $\mathrm{Si}_{3} \mathrm{~N}_{4}$ layer, but can be easily achieved with an $\mathrm{SiO}_{2}$ layer. In this case the SPP propagation constant is close to the one of a photonic quasi-TM mode in a Si nanowire, see the green colored area in Fig. 4(a). Therefore, in spite of its large FoM for the $\mathrm{Si}_{3} \mathrm{~N}_{4}$ dielectric, the subsequent discussions are based on a $\mathrm{SiO}_{2}$ layer.

\subsection{Three-dimensional structure}

The two-dimensional (2D) model is helpful for the general understanding of the operation principles and for a first device optimization. In this section we perform a full vectorial eigenmode analysis of a realizable three-dimensional (3D) SPPAM structure as outlined in Fig. 1. It consists of a $\mathrm{Ag} / \mathrm{ITO} / \mathrm{SiO}_{2} / \mathrm{Ag}$ multilayer structure on a $\mathrm{SiO}_{2}$ substrate. To ensure a SPP confinement along the $x$-axis, the top Ag layer has a finite width $w_{\text {SPP. }}$ It will be shown that the $2 \mathrm{D}$ results from Section 3.1 are still valid for the $3 \mathrm{D}$ case.

The lateral cross section of the plasmonic active part guiding the highly confined plasmonic mode is represented in Fig. 5. The choice of the configuration is such that efficient SPP excitation is guaranteed and fabrication is feasible with state-of-the-art nanofabrication techniques. As in the $2 \mathrm{D}$ case, SPP of such a structure exhibit a large field confinement within the ITO layer (see Fig. 5). In addition to the dielectric and the ITO thickness some other geometrical 3D properties come into play, such as the width of the top silver strip $w_{\mathrm{SPP}}$ and the thickness of the silver layer $h_{\mathrm{Ag}}$ which is chosen sufficiently thick to avoid SPP coupling to the top side of the upper silver strip and to ensure that the dispersion relation derived in Section 3.1 is still valid. The eigenmodes of the $3 \mathrm{D}$ configuration have been simulated with COMSOL Multiphysics. The simulations have been performed with rounded metal corners

\#143707 - \$15.00 USD Received 7 Mar 2011; revised 14 Apr 2011; accepted 16 Apr 2011; published 21 Apr 2011

(C) 2011 OSA

25 April 2011 / Vol. 19, No. 9 / OPTICS EXPRESS 8864 
having a radius of curvature $4 \mathrm{~nm}$. The propagation constant and length of the structure are plotted in Fig. 6 with a comparison to the 2D slab version of the structure discussed in the previous subsection.

Small deviations of the SPP propagation constant and propagation length in the case of the 3D structure are obviously caused by the finite values of $w_{\mathrm{SPP}}$ and $h_{\mathrm{Ag}}$. Moreover, it can be seen that for relatively large values of $w_{\mathrm{SPP}}$ the propagation constant of SPP in the 3D structure approaches the one of the slab structure. As opposed to the results for the propagation constant much larger differences can be seen in the propagation length when going from the 2D slab structure to the 3D plasmonic waveguides. The larger propagation length in the case of the 3D structure is due to the decrease of the field confinement in the ITO layer. Therefore, one can assume that the required modulator length in the 3D structure might be slightly longer
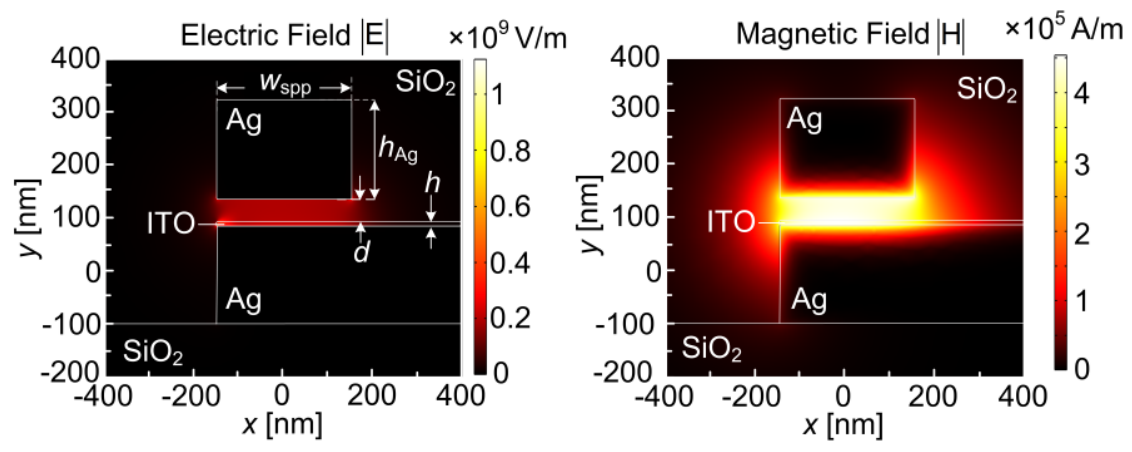

Fig. 5. SPP mode profile in terms of dominating magnetic and electric field components at the active part of the modulator: $\mathrm{Ag} / \mathrm{SiO}_{2}(40 \mathrm{~nm}) / \mathrm{ITO}(8 \mathrm{~nm}) / \mathrm{Ag}$. The fields are normalized to a cross-section power of $1 \mathrm{~W}$. Similar to the slab structure, $h$ and $d$ define the ITO and dielectric thicknesses, respectively. In addition, $h_{\mathrm{Ag}}$ and $w_{\mathrm{SPP}}$ describe the thickness and the width of the top silver electrode.

than the values predicted by the $2 \mathrm{D}$ theory. However, both propagation constant and propagation length approach their 2D equivalents for small dielectric thicknesses. The latter is a consequence of a decrease of the $d / w_{\mathrm{SPP}}$ ratio. The SPP waveguide width $w_{\mathrm{SPP}}$ is an additional degree of freedom which can be used for manipulating both propagation constant and propagation length.
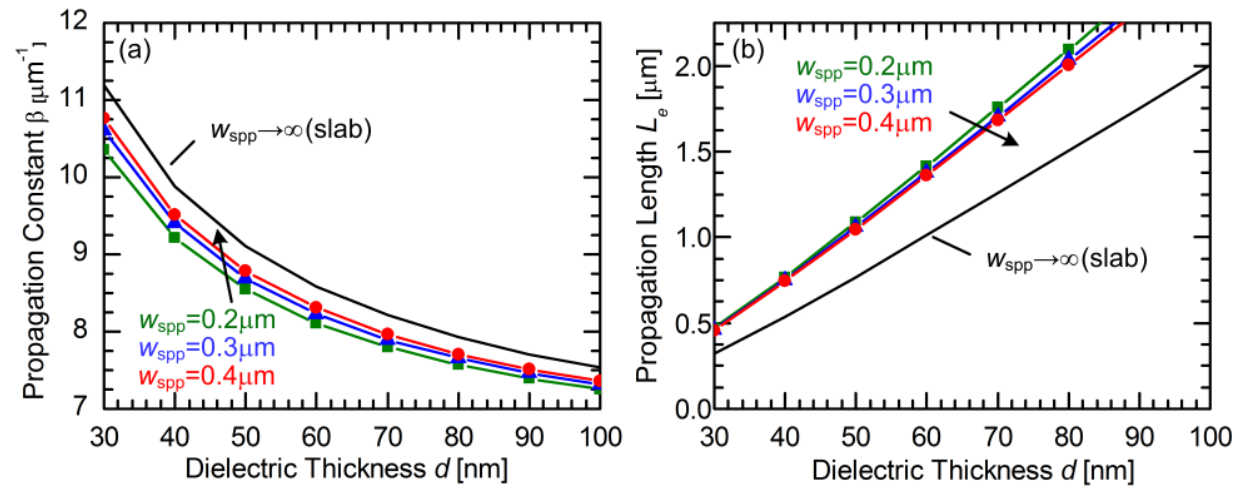

Fig. 6. Influence of plasmonic 3D waveguide width $w_{\mathrm{SPP}}$ on propagation constant (a) and propagation length (b). For comparison, the 2D results from solving Eq. (4) are added (black line). 
With a numerical 3D solution we have shown that the field can be horizontally confined along the $x$-axis under a silver bar with a finite width $w_{\text {spp }}$, Fig. 5. In such a three-dimensional waveguiding structure, all the requirements for an SPP modulation are fulfilled, namely high electric field strength in the dielectric layer, and optical field confinement in the ITO layer. The modulation speed is $R C$-limited, and estimate of this limiting frequency will be given next. The structure Fig. 5 having a cross section width of $300 \mathrm{~nm}$ and a length of $2 \mu \mathrm{m}$ has a capacitance of $C=0.7 \mathrm{fF}$. Assuming an internal generator impedance of $R=50 \Omega$, this results in a typical $R C$ time constant of $\tau=R C=35 \mathrm{fs}$. Therefore, letting aside problems of impedance matching, the modulation bandwidth is expected to be in the THz-region.

\section{Proof of principle}

In a proof-of-principle experiment we performed modulation measurements using a structure similar to the one proposed in [19]. The modulator differs, however, from the one suggested in this reference in that the electrodes are $61 \mu \mathrm{m}$ apart rather than $30 \ldots 50 \mathrm{~nm}$ as proposed in Fig. 5. The enormous distance between the electrodes in this test device results in a very low electric field in the metal oxide layer and consequently in a small extinction ratio. However, the structure is simple to fabricate and enables us to give a first proof of principle.

A scanning electron microscope picture of the fabricated device before lifting off the silver (upper-left triangle outlined in green) is shown in Fig. 7(b). For the fabrication of the device we used a standard silicon-on-insulator wafer with a $220 \mathrm{~nm}$ thick silicon layer on a $2 \mu \mathrm{m}$ thick buried oxide. Silicon wire waveguides of $240 \mathrm{~nm}$ (and $400 \mathrm{~nm}$ ) width and $220 \mathrm{~nm}$ height were fabricated using DUV lithography and standard CMOS processing. Subsequently, silicon nitride with a $200 \mathrm{~nm}$ thickness was sputter-deposited. The ITO (10 nm) / Ag (250 nm) layers were then deposited on top of the $\mathrm{Si}_{3} \mathrm{~N}_{4}$ layer via $\mathrm{RF}$ magnetron sputtering and electron beam evaporation, respectively. With photolithography the $10 \mu \mathrm{m}$ long device was transversely structured along the silicon waveguides. A lift-off process removed the remaining silver. To decrease the spacing between the electrodes, the back of the wafer was partially thinned by wet etching with potassium hydroxide $(\mathrm{KOH})$. In the etched-out area the silicon wafer was reduced to a thickness of $\sim 60 \mu \mathrm{m}$. With electron beam evaporation a $500 \mathrm{~nm}$ thick electrical contact was then formed at the bottom of the silicon substrate.

Experiments were performed by applying an electrical signal with $10 \mathrm{~V}$ peak-to-peak amplitude and a rectangular shape. This modulation signal was provided by a function generator with variable frequency and an internal impedance of $50 \Omega$. TM polarized monochromatic light at a wavelength of $1.55 \mu \mathrm{m}$ from a tunable laser source was coupled to the silicon strip waveguide via a lensed fiber. The resulting amplitude modulation of the optical signal was detected with an InGaAs photodetector connected to a lock-in amplifier. In order to subtract coherent electrical input-output crosstalk, the lock-in amplifier was calibrated with the DUT not being connected to the function generator.

Results of measured extinction ratios for modulation frequencies of up to $1.8 \mathrm{MHz}$ are shown in Fig. 7(c) for waveguides with $400 \mathrm{~nm}(\square)$ and $240 \mathrm{~nm}$ widths (•), respectively. Measured extinction ratios are small but clearly detectable. The drop in the extinction ratio when increasing the modulation frequency is due to the low-pass characteristic of the device (RC limiting frequency $\sim 58 \mathrm{kHz}$ ), see the equivalent circuit in the inset of Fig. 7(a). The capacitor $C_{1}=1.9 \mathrm{pF}$ describes the layers between top metal and silicon substrate, while $C_{2}=0.2$ $\mathrm{pF}$ describes the silicon substrate itself. The silicon strip has such a large resistance that it can be represented by a capacitor. The capacitance values were calculated using the well known dimensions and dielectric constants of the materials. The value of the resistance $R_{2}=1.2 \mathrm{M} \Omega$ was calculated from the resistivity of the silicon wafer (material data provided by the manufacturer). With this model the voltage drop $U$ across $C_{1}$ has been simulated with a conventional circuit simulator.

\#143707 - \$15.00 USD Received 7 Mar 2011; revised 14 Apr 2011; accepted 16 Apr 2011; published 21 Apr 2011

(C) 2011 OSA

25 April 2011 / Vol. 19, No. 9 / OPTICS EXPRESS 8866 
This voltage $U$ is then used for calculating the electric field in the $\mathrm{Si}_{3} \mathrm{~N}_{4}$ layer and at the ITO $/ \mathrm{Si}_{3} \mathrm{~N}_{4}$ interface thereby defining the boundary condition Eq. (12) that is needed to solve Poisson's Eqs. (9)-(11). The resulting induced carrier density $N_{i}$ of the ITO layer enters the Drude model Eq. (13) together with the parameters of Table 1. With $\varepsilon_{\text {ITO }}$ of the ITO layer known, we employ the dispersion relation for a three-layer structure (equivalent to Eq. (4) for a four-layer device) and find the complex propagation constant $\beta$, the imaginary part of which gives the absorption coefficient $\alpha_{\text {on }}\left(\alpha_{\text {off }}\right.$ for $\left.U=0\right)$. The difference $\left|\alpha_{\text {off }}-\alpha_{\text {on }}\right|$ defines the extinction ratio according to Eq. (6). In an iterative manner we modify $\omega_{\mathrm{p}}$ and $\gamma$ of Table 1 , until we reach a match for the measured ER.

A good match of the theoretically derived frequency response (solid blue line) and the experimental values $(\square, \bullet)$ has been found for an ITO plasma frequency $\omega_{\mathrm{p}}=0.8 \times \omega_{\mathrm{p} 0}$ and a collision frequency $\gamma=2.3 \times \gamma_{0}$, where $\omega_{\mathrm{p} 0}$ and $\gamma_{0}$ are published data [25], Table 1. Our values for the ITO plasma frequency and collision frequency are red shifted and blue shifted, respectively, relative to the values given in Table 1. Such a red shift of the plasma frequency and a blue shift of the collision frequency has been previously reported for ultrathin ITO layers [26].
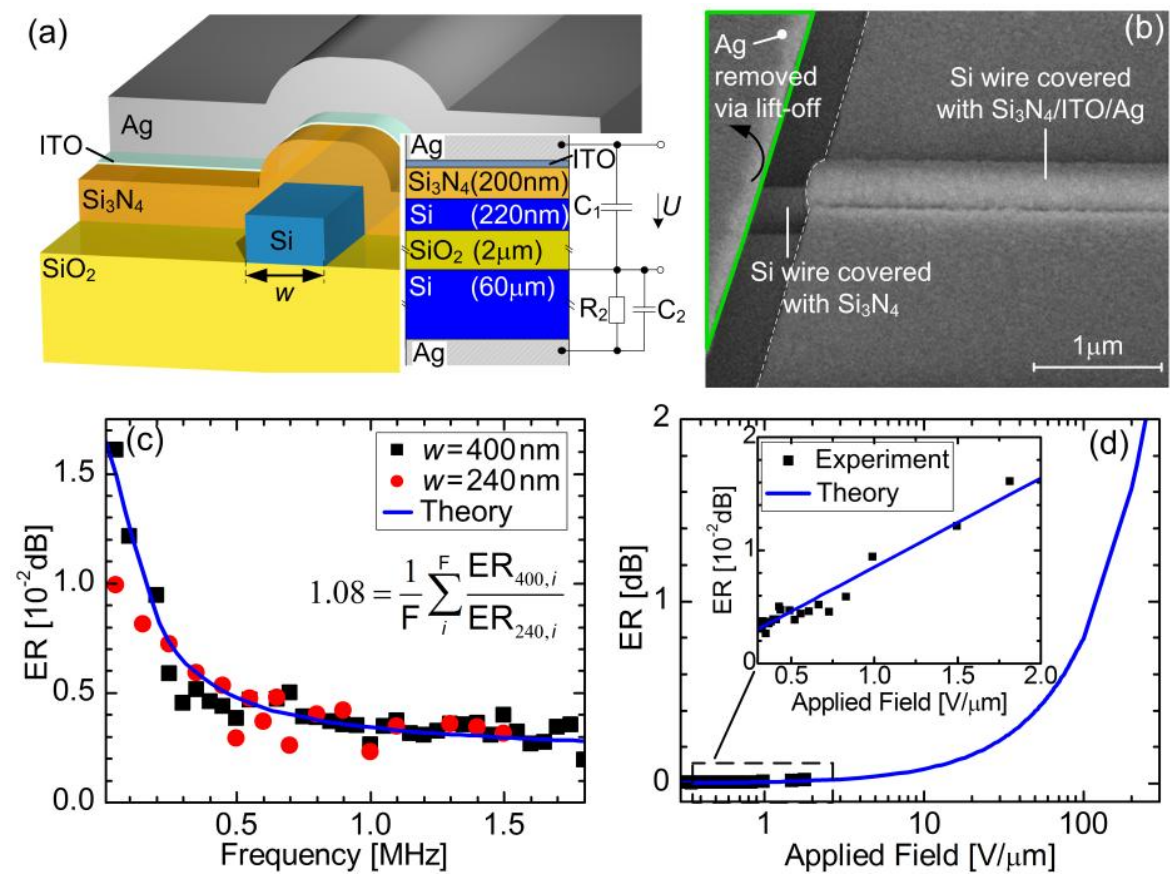

Fig. 7. The modulator structure for a proof-of-principle experiment together with the measured extinction ratios versus modulation frequency. (a) 3D schematic of the fabricated device and its lumped element model describing the low-pass characteristic of the device. (b) Scanning electron microscope picture of the device before lift-off (the area taken inside the green contour has been removed after completing the lift-off process). The device length is $L=10 \mu \mathrm{m}$ with an ITO thickness of $10 \mathrm{~nm}$ [19]. (c) Measured extinction ratios as a function of the frequency of the driving electrical signal. It can be seen that the extinction ratio does not depend on the silicon width $w$. The extinction ratio calculated by the theory is represented as a blue line with the fit parameters $\omega_{\mathrm{p}}=0.8 \times \omega_{\mathrm{p} 0}$ and $\gamma=2.3 \times \gamma_{0}$, where $\omega_{\mathrm{p} 0}$ and $\gamma_{0}$ are plasma and collision frequencies of ITO from Table 1. (d) Predicted ER for the strong electric fields obtained from the theory using fit parameter $\omega_{\mathrm{p}}$ and $\gamma$. 
To prove that the detected small amplitude modulation is a result of the carrier modulation in the ITO film and that it does not originate from plasma or thermal effects in silicon, we performed additional measurements.

To exclude the potential influence of a plasma effect in silicon we measured the extinction ratios for silicon wire waveguides with two different widths $w$. Both silicon waveguides were covered with a $200 \mathrm{~nm}$ thick silicon nitride layer, see Fig. 7(a). An eigenmode analysis revealed that the confinement factor of the quasi-TM mode is approximately two times larger for the $400 \mathrm{~nm}$ waveguide compared to the $240 \mathrm{~nm}$ waveguide. If the modulation was caused by the plasma effect in silicon, the measured extinction ratios for the wide and the narrow waveguide should be related by $2: 1$. According to Fig. 7(c) and its formula inset this is not true: The average measured quotient of the two extinction ratios is 1.08 , hardly larger than 1 and far away from 2 .

Next, a potential thermal effect due to heating of the sample while applying a voltage has been considered. Heat could be dissipated in resistor $R_{2}$ of the equivalent circuit Fig. 7(a). However, more heat would be dissipated at larger frequencies where a higher voltage drops across $R_{2}$ (as can be seen from the solid line in Fig. 7(c) which shows that $U$ decreases with frequency where the voltage between the silver electrodes is kept constant). As a consequence we would see a hypothetically larger extinction ratio at higher frequencies. However, according to our measurements in Fig. 7(c) this is not true, so we exclude dominant thermal effects.

The final question to be answered is: What could be expected for the optimized structure Fig. 1 where most of the voltage drops across the insulating $\mathrm{SiO}_{2}$ layer? To this end we plot the extinction ratio as a function of the modulating field strength as described before Fig. 7 . The inset Fig. 7(d) shows the measured values ( $)$ and the fitted curve (-). The main graph Fig. 7(d) then shows the ER prediction in a wider range of electric field strengths. A $1 \mathrm{~dB}$ extinction ratio can be obtained with an electric field of $100 \mathrm{~V} / \mu \mathrm{m}$ applied to a $10 \mu \mathrm{m}$ long device. Such an electric field strength can be easily achieved in the structure Fig. 1. This field strength is far below the dielectric strength which is in the order of $10^{3} \mathrm{~V} / \mu \mathrm{m}$ for materials like $\mathrm{SiO}_{2}$ and $\mathrm{Si}_{3} \mathrm{~N}_{4}$.

\section{Conclusion}

We investigated an electro-optic surface plasmon polariton absorption modulator (SPPAM) operating at the $1.55 \mu \mathrm{m}$ telecommunication wavelength. The underlying principles were analyzed. A three-layer prototype was designed, and first proof-of-principle experiments were performed. The device is $R C$-limited with a typical $R C$ time constant of $\tau=35 \mathrm{fs}$. Therefore, the theoretical modulator speed is in the THz-region thus fulfilling future ultra-high speed data modulation requirements. For an optimized four-layer modulator with $\mathrm{SiO}_{2}$ dielectric, a 1 $\mathrm{dB}$ extinction ratio has been predicted for a device length of $2 \mu \mathrm{m}$ and a total plasmonic loss in the range of $18 \mathrm{~dB}$. However, for the same extinction ratio of $1 \mathrm{~dB}$ the device length can be significantly decreased by utilizing $\mathrm{Si}_{3} \mathrm{~N}_{4}$. With a modulation field strength of $250 \mathrm{~V} / \mu \mathrm{m}$ the loss would amount to $12 \mathrm{~dB}$ for a device length of about $0.5 \mu \mathrm{m}$ which is comparable to the footprints of electronic devices.

\section{Appendix A}

In this appendix, we discuss the material parameters of ITO and silver used in the above calculations.

\section{A.1 Material parameters of ITO and Silver}

The frequency dependence of the dielectric permittivities of both silver and ITO are taken into account by the Drude model,

\#143707 - \$15.00 USD Received 7 Mar 2011; revised 14 Apr 2011; accepted 16 Apr 2011; published 21 Apr 2011

(C) 2011 OSA

25 April 2011 / Vol. 19, No. 9 / OPTICS EXPRESS 8868 


$$
\varepsilon=\varepsilon_{\infty}-\frac{\omega_{\mathrm{p}}^{2}}{\omega^{2}+i \omega \gamma} .
$$

In the infrared spectral region, the contribution of the bound electrons is included in the frequency independent parameter $\varepsilon_{\infty}$. The plasma frequencies $\omega_{\mathrm{p}}$ needed in the Drude model are given in Table 1. For silver, the plasma frequency is $9.12 \mathrm{eV}$ [27]. For comparison, the Drude model parameters for an $8 \mathrm{~nm}$ thick ITO layer are also listed.

Table 1. The Drude Parameters of ITO and Ag

\begin{tabular}{lccc}
\hline \multicolumn{1}{c}{ Parameters } & $\mathrm{ITO}_{\text {off }}$ & $\mathrm{ITO}_{\mathrm{On}}\left(U=10 \mathrm{~V} / 30 \mathrm{~nm}\left(\mathrm{SiO}_{2}\right)\right)$ & $\mathrm{Ag}$ \\
\hline $\begin{array}{l}\text { Background permit- } \\
\text { tivity } \varepsilon_{\infty}\end{array}$ & 3.9 & 3.9 & 1 \\
\hline $\begin{array}{l}\text { Plasma frequency } \omega_{\mathrm{p}} \\
\text { Collision frequency }\end{array}$ & $2.9 \times 10^{15} \mathrm{~s}^{-1}[25]$ & $2.914 \times 10^{15} \mathrm{~s}^{-1}$ & $1.38 \times 10^{16} \mathrm{~s}^{-1}$ \\
$\gamma$ & $1.8 \times 10^{14} \mathrm{~s}^{-1}[25]$ & $1.8 \times 10^{14} \mathrm{~s}^{-1}$ & $3.22 \times 10^{13} \mathrm{~s}^{-1}$ \\
\hline $\begin{array}{l}\text { Electron effective } \\
\text { mass } m_{\text {eff }}\end{array}$ & $0.35 \times \mathrm{m}_{\mathrm{e}}[24]$ & $0.35 \times \mathrm{m}_{\mathrm{e}}$ & $1 \times \mathrm{m}_{\mathrm{e}}$ \\
\hline $\begin{array}{l}\text { Carrier density } N_{0} \\
\begin{array}{l}\text { Complex dielectric } \\
\text { permittivity }\end{array}\end{array}$ & $9.25 \times 10^{26} \mathrm{~m}^{-3}$ & $\left\langle N_{i}(y)\right\rangle_{h=8 \mathrm{~nm}}=9.34 \times 10^{26} \mathrm{~m}^{-3}$ & $6.03 \times 10^{28} \mathrm{~m}^{-3}$ \\
\hline
\end{tabular}

Different values have been reported for the plasma frequency of ITO. They are mostly derived by observing SPP resonances in the near - infrared region. The plasma frequency values used in this paper are taken from Michelotti et al. [25]. They investigated SPP resonances for several commercially available ITO samples with various thicknesses. Using these data they calculated and reported on plasma and collision frequencies of ITO by fitting experimentally obtained transmittance and reflectance spectra with the ones calculated through the Drude model. Observing the plasmon resonances for ITO films with various thicknesses in the wavelength range from $1.4 \mu \mathrm{m}$ to $2.0 \mu \mathrm{m}$, Rhodes et al. [28] reported significantly larger plasma frequencies. Moreover, they found that the plasma frequency is constant for samples with thicknesses down to $30 \mathrm{~nm}$.

On the other hand, Dasgupta et al. [26] investigated ITO ultrathin films, and the absolute values of plasma frequencies reported by them is approximately two times smaller than the ones shown in Table 1. However, based on these findings and other experimental investigations [26,29], one may conclude that for ITO layers thicker than $8 \mathrm{~nm}$ the free carrier density of ITO saturates towards its bulk value. Thus, the plasma frequency of ITO used in this paper is the one from Ref. [25]. which is near the average of the values so far reported in the literature. It should be emphasized that since the ITO thicknesses of interest are in the range of 8 $\mathrm{nm}$ to $15 \mathrm{~nm}$, it is possible to optimize the free carrier density by varying the thickness of ITO such that bound SPP strongly confined in ITO film exists at the wavelength of $1.55 \mu \mathrm{m}$. Moreover, the use of the parameters given above is justified by the fact that the key material parameter influencing the overall modulator performance is mainly the plasma frequency of ITO which can be optimized by controlling the ITO sputtering process via the oxygen content, and by the tin-to-indium ratio as well as the temperature and pressure in the deposition chamber.

\section{Acknowledgements}

We acknowledge support by the DFG Center for Functional Nanostructures (CFN) and the Karlsruhe School of Optics and Photonics (KSOP). 